\title{
Supporting Social Innovation through Visualisations of Community Interactions
}

Daniel Rough

Stefano De Paoli

Francesco Botto

(C) Owner/Authors | ACM 2019. This is the authors' version of the work. It is posted here for your personal use. Not for redistribution. The definitive Version of Record was published in Proceedings of 9th International Conference on Communities \& Technologies (C\&T 2019), available at: http://dx.doi.org/10.1145/3328320.3328371 


\section{Supporting Social Innovation through Visualisations of Community Interactions}

\author{
Daniel Rough \\ Abertay University \\ Dundee, United Kingdom \\ d.rough@abertay.ac.uk
}

\author{
Stefano De Paoli \\ Abertay University \\ Dundee, United Kingdom \\ s.depaoli@abertay.ac.uk
}

\author{
Francesco Botto \\ Fondazione Bruno Kessler \\ Trento, Italy \\ fbotto@fbk.eu
}

\begin{abstract}
Online communities that form through the introduction of sociotechnical platforms require significant effort to cultivate and sustain. Providing open, transparent information on community behaviour can motivate participation from community members themselves, while also providing platform administrators with detailed interaction dynamics. However, challenges arise in both understanding what information is conducive to engagement and sustainability, and then how best to represent this information to platform stakeholders. Towards a better understanding of these challenges, we present the design, implementation, and evaluation of a set of simple visualisations integrated into a Collective Awareness Platform for Social Innovation platform titled commonfare.net. We discuss the promise and challenge of bringing social innovation into the digital age, in terms of supporting sustained platform use and collective action, and how the introduction of community visualisations has been directed towards achieving this goal.
\end{abstract}

\section{CCS CONCEPTS}

- Human-centered computing $\rightarrow$ Social network analysis; Visual analytics; Computer supported cooperative work;

\section{KEYWORDS}

Social network analysis, information visualisation, social science

\section{ACM Reference Format:}

Daniel Rough, Stefano De Paoli, and Francesco Botto. 2019. Supporting Social Innovation through Visualisations of Community Interactions. In Proceedings of 9th International Conference on Communities \& Technologies (C\&T 2019). ACM, New York, NY, USA, 11 pages. https://doi.org/10.1145/ nnnnnnn.nnnnnnn

\section{INTRODUCTION}

The effectiveness of visualisation for communicating large and complex information sources has prompted its widespread use in non-technical domains, allowing diverse sets of users to make sense of their data. In light of this, visual representations of the rich information generated by community-driven digital platforms could provide novel insight into the dynamics of these communities for their stakeholders. However, the design and application

Permission to make digital or hard copies of part or all of this work for personal or classroom use is granted without fee provided that copies are not made or distributed for profit or commercial advantage and that copies bear this notice and the full citation on the first page. Copyrights for third-party components of this work must be honored.

For all other uses, contact the owner/author(s).

C\&T 2019, June 2019, Vienna, Austria

(C) 2019 Copyright held by the owner/author(s)

ACM ISBN 978-x-xxxx-xxxx-x/YY/MM.

https://doi.org/10.1145/nnnnnnn.nnnnnnn of visualisations in such a context requires an understanding of community goals that are very often social and ethical in nature. Thus, a design that supports such goals must be carefully adapted to this context of use. Even when visualisations have been used in community-building processes, often these are scientific or technical in nature [24, 27]. Conversely, platforms for social innovation are composed of end-users who are not necessarily able to interpret complex representations. However, the interactions on these platforms are a rich complex network, insights of which need to be effectively communicated to its members, platform administrators and external entities with a stake in the social innovation process.

Visualisations for participatory action have been investigated by Schoffelen et al., whose challenges in engaging and informing passers-by with visual representations in public spaces closely align with the challenges of digital visualisations for community-driven platforms [34]. As well as ensuring that a visualisation solution can be easily interpreted by non-technical users, it must also allow users to trace the 'backstories' behind the representation, to understand the social objectives of community members. With a goal of understanding how these requirements can be satisfied, this paper describes the design, implementation and evaluation of visualisations in commonfare.net, a community-driven digital social innovation project. The visualisations are based on metrics derived from social network analysis and provide relevant information to three different stakeholder groups - community members, platform administrators, and external entities with an interest in commonfare.net. The visualisations, and the information that they represent, have been refined through the following two research questions:

(1) What information can be derived from social network analysis to support the development of communities?

(2) How can this information be most effectively visualised to these communities while accounting for user privacy?

The paper is organised as follows: we first provide an overview of collective awareness platforms in general, with specific details of commonfare.net, and relevant literature on social network analysis and visualisation. We then present the goal of the project's different visualisations, and discuss results that have emerged thus far. We conclude with some reflections for future work.

\section{BACKGROUND}

Our work on community visualisations is just one facet of the commonfare.net platform and its ethos of promoting social innovation. In this section, we provide a brief overview of social innovation and similar collective awareness platforms, with a focus on core 
features of our work with commonfare.net - social networks and their analysis/visualisation.

\subsection{Social Innovation and CAPS}

A report from the European Commission formally defines social innovation as "new ideas (products, services and models) that simultaneously meet social needs (more effectively than alternatives) and create new social relationships or collaborations." [9, p. 9]. Consequently, digital social innovation has emerged as the application of technologies (such as open hardware and open-source software) to foster social change and increase social collaboration in areas that are not provided by traditional welfare state, thereby enhancing societal capacity. Digital social innovation platforms are complex socio-technical systems with two core strategies: a "bottom-up" approach that enables individuals to harness collective action, and a focus on the use of technology to solve emerging social problems.

commonfare.net is one of many Collective Awareness Platforms (CAPS) funded by the European Commission, which use digital social innovation to raise awareness of various issues and offer sociotechnical solutions, allowing affected groups to share resources and take collaborative action [10]. Bellini et al. describe, categorise, and assess the impact of initially funded CAPS projects, which exemplify platforms closely related to commonfare.net [4]. Examples include: Open4Citizens [28], which aims to enable communities to understand and collectively manage open data towards the creation of public services, and PROFIT [17], which provides a resource for raising financial awareness and increasing financial capability of its users. While differing in their domain and scale, such CAPS projects have various commonalities:

- a goal to raise awareness of a societal issue and generate collective knowledge on this issue across communities

- a strategy for encouraging target community members to engage in platform knowledge contribution

- a means for project partners and external organisations to obtain insight from platform engagement

The backgrounds of target communities, types of contribution afforded and requirements of platform sustainability all determine how these common features are represented in a CAPS platform.

\subsection{An overview of commonfare.net}

Here we offer a brief overview of the commonfare.net platform and its aims, and describe the socio-political ethos and approach that characterise the platform. commonfare.net is a CAPS project that seeks to support people who are experiencing poverty and precarity in Europe, such as young people who are no longer in the education system and face difficulties finding a job. Due to the 2008 global financial crisis, which led to a weakening of state-based approaches to welfare, social innovation can help by favouring bottom-up emancipation and empowerment processes for those who have become poor as a result. Hence, commonfare.net has the ambitious aim to offer tools that can support these "new poors" to improve their living conditions. The platform has been developed through participatory design research involving people from three different countries, specifically: unemployed and precarious young people in Croatia, precarious workers in Italy and the Netherlands and, finally, non-Western migrants and benefit recipients in the Netherlands [6].

The platform seeks to promote and facilitate commonfare, an alternative approach to social welfare [15]. A commonfare approach is grounded in the recognition that social and economic spheres are not separate, but instead are inextricably and intricately connected. Key features of a commonfare approach include: the reappropriation of the common by communities (a common that encompasses both immaterial as well as material goods), provision of a Basic Income to all members of society and the development of complementary financial circuits for the management and circulation of social wealth.

Users of the platform are called "Commoners", a name that was chosen in order to emphasise the focus that the platform has on creating a common and a thriving community that can benefit from this common. Thus, commonfare.net seeks to facilitate cooperation among its users, who share resources in a bottom-up welfare process, for their common good. Core components of the platform are described as follows:

- Users can benefit from a digital currency system - a digital token called "commoncoin". Each user has a personal wallet of commoncoins, which are distributed as a form of monthly basic income, and can be transferred to other users.

- Users can exchange skills, goods and services, facilitated by the use of the commoncoin wallets, through a section of the platform entitled Commonplace.

- Users can utilise storytelling functionality for sharing positive experiences and good practices for tackling new forms of poverty. Storytelling is seen as an important way of creating a common of knowledge and providing stories of emancipation from poverty conditions.

- Each registered user has a profile, which includes basic details such as her Commoner name and picture, as well as her platform "reputation", which acts as a measure of how much the user contributes to the common good of commonfare.net.

commonfare.net places particular emphasis on storytelling and on the sharing of experiences. The platform is designed in a way that users can write about their own experiences (whether positive or negative), ways of dealing with their own conditions (e.g., precarious work or unemployment), or innovative processes they have put in place to help themselves and other people. For example, the platform includes stories related to experiences of multiculturalism and cooperation, workers' self-organising activities, community shops, recycling and the use of recycled material for creating small sustainable businesses, to name a few. The overarching concept is that stories are also a way of interacting with others - users of commonfare.net can engage with story creators through public comments or private conversations, with a view to learn more or to reproduce certain experiences in their own contexts. Further, users can donate their commoncoins to story authors as a way of showing appreciation for sharing the story.

In the context of the commonfare.net platform and its community, this paper reports on activities conducted to support community social innovation development, through the design and 
evaluation of a series of visualisations. These serve both Commoners as well as community managers, by representing metrics of interest to each group, derived from Social Network Analysis.

\subsection{Social Network Analysis}

Rooted in sociology, the study of relationships between individuals, the strength and directionality of ties that bind them together, and the properties of the emergent network of relationships, has enabled insights into social structure beyond the capacity of individualistic methods [39]. Concepts from mathematical graph theory have been employed to provide quantitative insights into community structures for sociologists and anthropologists [30]. In practical terms, these insights can be employed to cultivate and sustain existing communities of users within an online platform.

Localised metrics that focus on single nodes or small groups can reveal the role of individuals within their social network and to what extent they affect others around them. Brown and Feng employ a graph decomposition algorithm to find highly influential users in a graph of Twitter interactions, determining those who act as propagators of information [7]. Network metrics may be used as an indicator of trustworthiness or "reputation", an approach used in Google's PageRank algorithm [32], which assigns a high reputation to web pages that are linked to by other high-reputation pages. When applied to social networks, reputation can be allocated to users themselves [2]. In platforms that employ such reputation systems, network metrics can highlight collusive behaviour. For example, Shen et al. present algorithms for collusion detection, which can minimise the risk of reputation misuse [35].

Global network metrics offer further insight into social structures beyond that of the individual; social networks exhibit a high level of clustering, or network transitivity, indicative of smaller communities that form close ties within the larger network structure. Fortunato presents a comprehensive review of work on graph-based community detection, including a survey of algorithms employed, and the applications of understanding such communities in different network types [14]. For social networks, Cao et al. introduce an improved algorithm for recommending content to a user based on preferences within their implicit community [8]. Papadopoulos et al. survey community detection in social media, highlighting its application to identifying topics of social interest and communities affected by particular events [33].

\subsection{Community visualisation}

While network analysis provides rich information on the structure and function of social networks, this information must be communicated effectively to be of practical value. In general terms, the effectiveness of an information representation can be considered as its ability to generate insights for its user [31]. Community visualisations can provide insights to administrators and researchers, related to the structure and function of the community itself. Further, insights at an individual level can be obtained by members through understanding their place within the larger structure.

2.4.1 Community insight. Social network visualisations have been applied as early as the 1930 s, when Jacob Moreno introduced simple "sociograms" for the analysis of small group interactions [29].
Since then, a variety of powerful tools for general graph visualisation have been made available for generating insight from large, complex, dynamic social structures. Wu et al. present a general review of visual analytics of social media data, including novel tools for understanding collective community behaviour [40]. In the social science domain, Ghani et al. focus on the visualisation of multimodal social networks, demonstrating the effectiveness of visualising correlations across communities for social scientists' analyses [18]. In the education domain, Dawson introduces a tool for visualising networks of students and teachers within a Virtual Learning Environment, allowing low-performing students to be identified and allocated further teacher support [11].

2.4.2 Personal insight. From the perspective of online community members themselves, transparent metrics on personal interactions, and those of others, are conducive to strengthened community engagement. Sun and Vassileva demonstrated that providing students with a visualisation of their contributions to a class paper-sharing community encouraged further participation [36]. Similarly, Gilbert and Karahalios obtained feedback from open-source developers on a visualisation of code project contributions, who expressed increased motivation from a community-wide display of their work [19]. Valkanova et al. evaluated a public social visualisation of energy usage, through which users engaged in critical thinking about their own energy spending, and that of others [37].

In addition to these global network visualisations, individuals benefit from representations that allow for critical reflection on their personal activities over time. Examples pertaining to social interactions include Vizster, which utilises the common node-link network representation to provide an egocentric view of the interacting user's social network [22], and Themail, which depicts individuals' email conversation history [38]. Through such personally focused visualisations, users engage in critical reflections about their place and history within a community.

\section{SOCIAL NETWORK METRICS}

Towards designing visualisations that aim to both motivate users of the commonfare.net platform to make contributions, and explicate important platform dynamics to administrators, we derived metrics through social network analysis methods. The commonfare.net platform data consists of various types of interactions between members, as well as their interactions with stories, listings and tags. This is extracted from the platform database in GEXF format $^{1}$ (a graph-based interchange format based on XML) which allows the history of platform interactions to be represented as a dynamic social network. This is then analysed using the Python NetworkX library ${ }^{2}$ to extract relevant network insights, and generate formatted output files for visualisation purposes. This section details the metrics of interest, and the underlying algorithms for their generation.

\subsection{A reputation metric - "commonshare"}

Our metric that represents an individual's contribution to commonfare.net, hereby known as commonshare, is primarily based on

\footnotetext{
${ }^{1}$ https://gephi.org/gexf/format/

${ }^{2}$ https://networkx.github.io/
} 
their core number within a $k$-core decomposition of the graph of interactions. A $k$-core is a maximal subgraph that contains nodes of degree $k$ or more (those that have $k$ or more edges) and the core number of a node is the largest value $k$ of a $k$-core containing that node. An example $k$-core decomposition is pictured in Figure 1, which shows that the central, densely connected yellow nodes have a core number of 3 , whereas the peripheral blue nodes have a core number of 1 . In general, a node's core number represents how central it is to the network.

This metric is important to both community members and administrators. For members, their commonshare represents a form of reputation, accrued through platform interactions, which can motivate participation and establish trust between members. For administrators, it is an indicator of overall platform activity, as well as particularly active members, whose high core number is an accurate measure of their strong influence in the network [7, 26].

We use the Python NetworkX implementation of the $k$-core decomposition algorithm introduced by Batagelj and Zaversnik [3], the pseudocode for which can be seen in Algorithm 1. It recursively prunes nodes of degree less than $k$ such that remaining nodes belong to a given $k$-core.

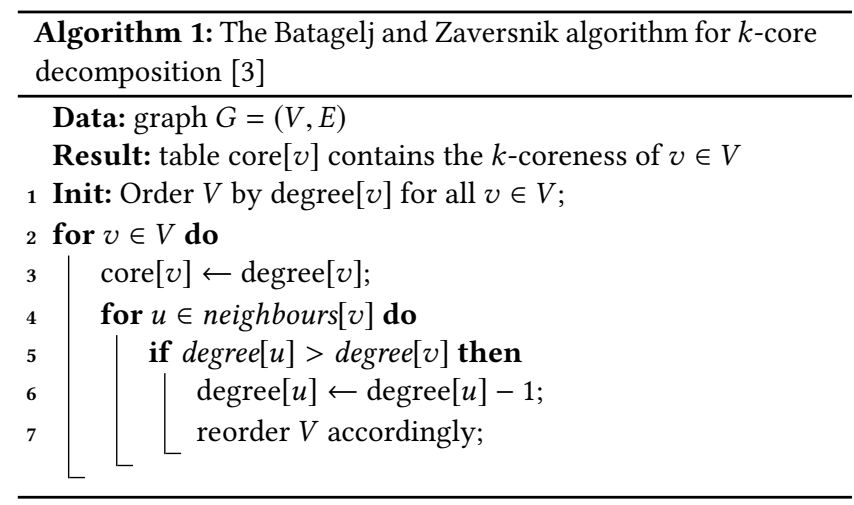

Further, we have made adjustments to account for different types of interaction, by augmenting a node's degree with the weight of its edges, as proposed by Garas et al [16]. Following insight from our network visualisation described in Section 5, users' commonshare is adjusted to give precedence to the weights of their interactions, rather than the frequency of these interactions. A node's 'augmented' degree is calculated as $k_{i}^{\prime}=\sqrt{k_{i}} \sum w_{i j}$, where $k_{i}$ is the node's original degree, and $\sum w_{i j}$ is the sum of its edge weights.

\subsection{Potential collusive behaviour}

Collusive behaviour is a prevalent issue in systems where reputation can be accrued $[1,35]$. In general, such behaviour is characterised by two or more users cooperatively boosting their reputation by providing multiple high ratings to each other, while ignoring or giving low ratings to other users. It is important for commonfare.net administrators to identify this behaviour so that any benefits associated with a high commonshare are allocated fairly.

We use a simplified implementation of the algorithm proposed in [35] to determine whether a significant proportion of a user's total reputation comes from another node, and whether that node

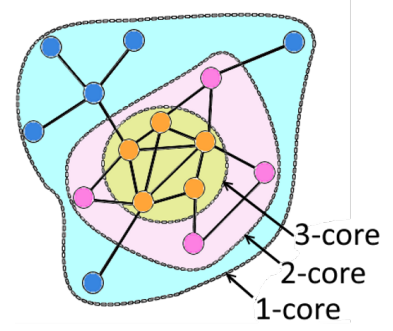

Figure 1: An example $k$-core decomposition

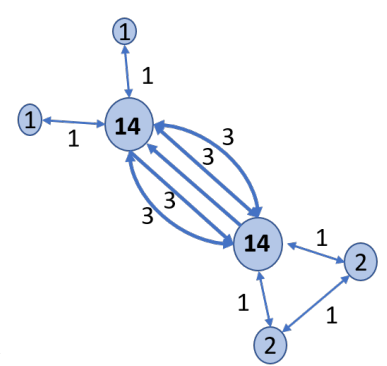

Figure 2: An example of potential collusive behaviour reciprocates. If this is the case, then these two nodes are flagged as potential colluders. For example, Figure 2 depicts two nodes that have multiple, weighted interactions with one another while interacting minimally with other nodes. Pseudocode for the simplified algorithm is given in Algorithm 2. The initial map A, which pairs nodes to their edges, is only populated with nodes that exceed a certain level of activity, to avoid checking all possible node combinations. Line 8 indicates that potentially collusive behaviour occurs when the ratio of edge weights from one node to the total sum of edge weights of an examined node exceeds a given threshold $T$. However, nodes are only considered colluders if this relationship is reciprocal (line 4).

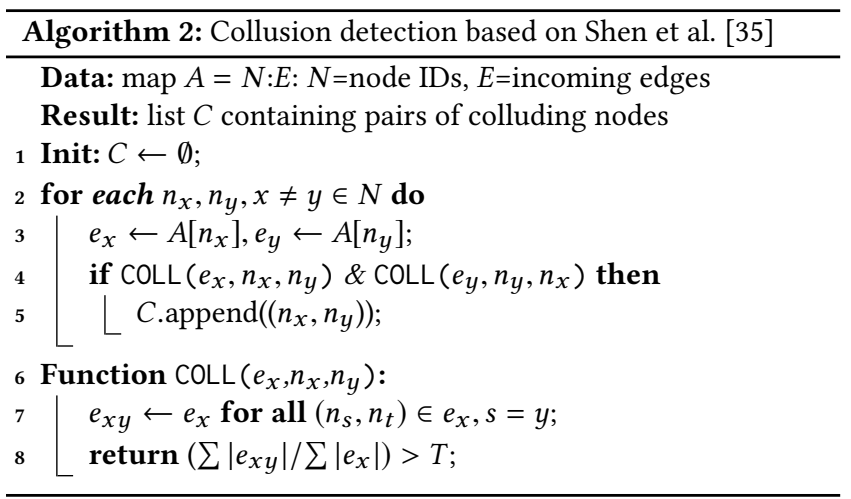

While this is a basic means of detection that does not account for more subtle forms of collusion, the platform is not at a scale where sophisticated, automated detection mechanisms must be put in place. Instead, this approach serves the purpose of highlighting potential anomalies to administrators for investigation. As the platform grows, additional options will be considered.

\subsection{Static and dynamic communities}

While community detection in static networks is well-studied [14], the temporal evolution of social networks, and thus the presence of communities at different points in time, is of greater interest from a commonfare.net administrator's perspective. The ability to observe the growth, shrinkage, beginning and end of dynamic communities over time affords insight into the stability of these communities, including users or stories that act as stable anchors, 
and temporal events that form or disrupt them. As an implementation, we use the solution proposed by Greene et al. of comparing communities across discrete time steps [20], which is independent of the static community detection algorithm used. At each time step, a set of static communities is generated, for which we use a Python implementation ${ }^{3}$ of the Louvain method of community detection, first proposed by Blondel et al [5]. Pseudocode for the approach is shown in Algorithm 3.

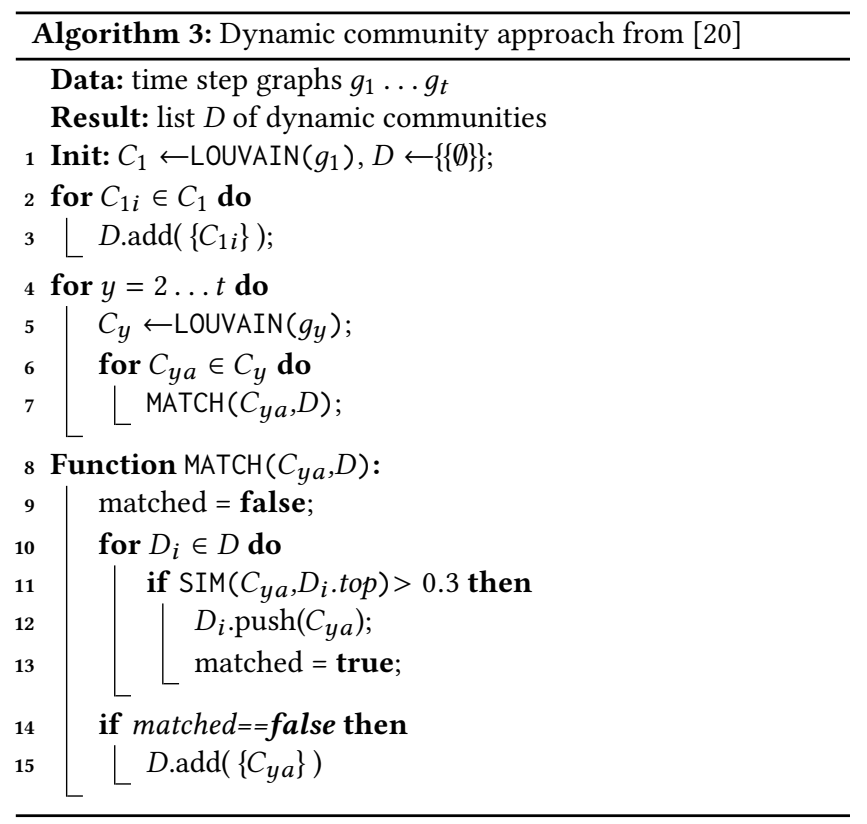

First, the initial list of static communities in the first time step of commonfare.net is computed. For every time step thereafter, static communities are again computed and compared to those discovered in previous time steps, using a comparison function (line 8). The similarity of each new static community to the most recent instance of each dynamic community is measured by the Jaccard similarity coefficient [23] (line 11). If the similarity is above a certain threshold (in this case 0.3 ) this static community is recognised as an instance of the dynamic community, and is appended onto it. If no match is found for the static community, it becomes the beginning of a new dynamic community (line 15). Varying the size of time step yields different results, with two weeks found to be a sensible value with respect to the platform activity on commonfare.net.

\section{DESIGN OF VIEWS}

We assert that the metrics derived in the previous section can be applied to stimulate participation, identify dynamic behaviour patterns, and discover topics of interest within communities of platform users. Towards effectively communicating these metrics, three stakeholder groups have been identified, namely: the registered users of commonfare.net who engage with each other through the platform's features; administrators of commonfare.net who have an active interest in sustaining the platform's growth; and visitors

\footnotetext{
${ }^{3}$ https://github.com/taynaud/python-louvain
}

to the commonfare net platform with an interest in community dynamics, from a social action or research perspective. For these three groups respectively, the following views have been developed:

(1) a network analysis view providing administrators with social network analysis insights - in particular, the development of the community and of potential manipulation of the commonshare reputation system

(2) a personal view integrated into user profiles, which commonfare.net users can inspect to understand their contributions to the platform, and those of others

(3) a public view intended for use by public authorities and researchers, showing current trends of story-telling and participation within the platform

Each view is implemented with the D3 Javascript library ${ }^{4}$. This section describes the initial design decisions undertaken for the views, with their typical use cases.

\subsection{Network analysis view}

Unlike platforms that connect existing offline communities, commonfare.net enables the formation of new, geographically disparate communities that, due to their potential fragility, need careful management to cultivate and sustain. As explained in Section 3, the following two elements are of interest to platform administrators:

(1) Community development. Identification of users central to the activity on commonfare.net is important to ensure the continued development of the platform. For example, such users are ideally suited for diffusion of important information through a network [7, 25], and particularly active users could be invited to participate in administrative roles in the network, increasing their sense of platform ownership.

(2) Manipulative activity. It is also to be expected, given the potential benefits of having a high reputation on commonfare.net, that users may try to increase their perceived contribution by creating meaningless content, or colluding with other users by repeatedly engaging in meaningless interactions $[1,35]$. Ensuring these users are not rewarded for such actions is necessary to keep commonfare.net fair and equal.

The overall network analysis view is shown in Figure 3. This section describes the labelled components and how they provide visual representations of important metrics.

4.1.1 Interaction graph. Figure 3A shows the interaction graph of commonfare.net as a node-link representation. Each node represents a user (blue), story (red), listing (purple) or tag (green), and each coloured link between these nodes represents an interaction, such as a conversation, transaction, or story comment. The size of each node represents its commonshare, and allows administrators to identify strong levels of participation at a glance. This view also highlights nodes in dark red if they are potentially involved in collusive activity. For example, Figure 4 shows two user nodes (U1, U2) who, while never interacting directly with each other, have accumulated commonshare by repeatedly interacting with each other's stories (S1, S2). A slider (A1) allows the user to view the graph of interactions for any two-week interval (the current level of granularity) in the platform's history, explicating the dynamics

\footnotetext{
${ }^{4}$ https://d3js.org
} 


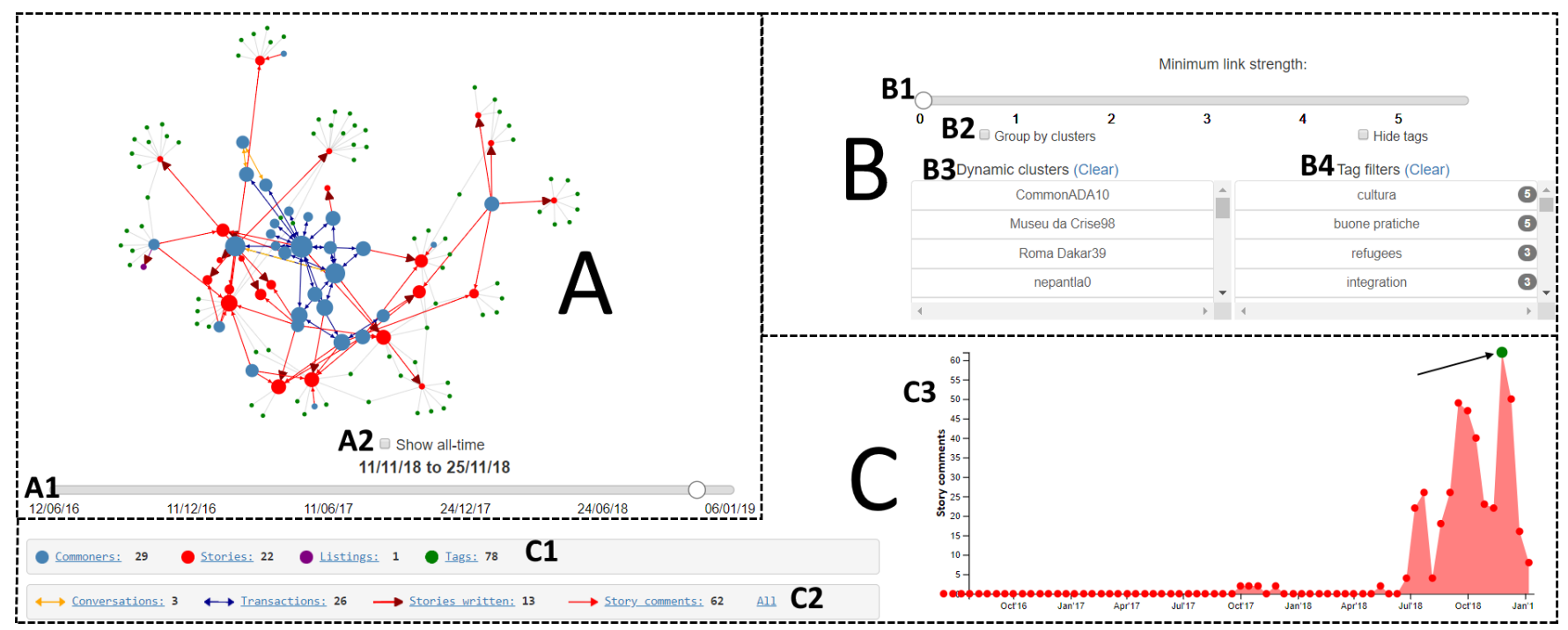

Figure 3: Network analysis view, segmented into three main components

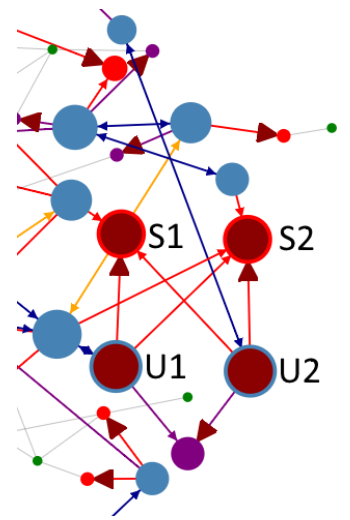

Figure 4: Detection of collusion through indirect interactions
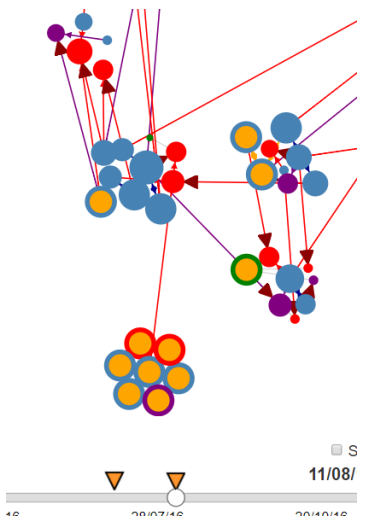

Figure 5: Members of dynamic communities at a single time instance

of platform activity. A checkbox (A2) toggles the cumulative view of all interactions, and thereby the most influential users over the history of commonfare.net.

4.1.2 Graph analysis widgets. Figure $3 \mathbf{B}$ contains widgets that can be used to manipulate the graph for more detailed analysis. A slider (B1) allows filtering of the graph by edge weight to highlight strong interactions, and a menu (B4) allows filtering by specific tags.

The representation of both static and dynamic communities, as derived in Section 3.3, involves further manipulation of the interaction graph. A checkbox (B2) transforms the graph layout to cluster nodes by their static community at any given time instance, and a menu (B3) displays a text list of dynamic community names. When a community is selected from this menu, all nodes that form part of this community are highlighted. Small markers appear on the timeline slider to indicate points of time where the dynamic community has been active. Figure 5 illustrates this idea - the bottom

cluster of yellow nodes represents a dynamic community at this time step. Other yellow nodes appear in different clusters because, in a past or future time step, they are also part of this community. The two markers on the timeline indicate two time periods that this community has been active in.

4.1.3 Temporal variations of simple metrics. Finally, Figure $3 \mathbf{C}$ highlights the components through which trends in various aspects of platform activity can be inspected. Basic figures for this two week period are shown, including the number of each type of node (C1) and the total number of each interaction type (C2). Clicking on one of these metrics will update an area chart (C3) to show how this metric has changed over time. In the illustrated example, the chart shows that story comments reached a peak during this period (highlighted by the indicated green dot on the chart).

4.1.4 Use cases. The effectiveness of this view is being continuously evaluated through its ongoing use by project partners, to be discussed in Section 5. However, we highlight further questions that can be answered as the platform grows:

- Who are the most engaged users? 'Key players' in the commonfare.net network are those who interact with many others and produce helpful, engaging content. Such users could be offered an administrative role in the platform, sustaining it beyond the project's lifespan.

- What is happening on the platform? In a general sense, the view allows a network administrator to understand how users are interacting, and whether actions can be taken, such as disrupting collusive behaviour, or facilitating contact between clusters of similar users

- What isn't happening on the platform? Conversely, it is important to determine a lack of activity, so that action towards promoting commonfare.net to different groups can be taken. 


\subsection{Personal view}

Originally, the concept of "commonshare" arose from participatory design research with potential users of commonfare.net, who wanted to represent their contributions in a non-competitive way. This research will be detailed in future work, but we highlight some key results. Foremost, existing reputation systems based on buying and selling of products (such as those on eBay and Amazon) are inappropriate to represent emotionally meaningful actions taken on commonfare.net. In particular, due to the likelihood of emotional and financial issues of commonfare.net users, a reputation system must avoid inciting feelings of negative judgment, or any form of indebtedness. Thus, instead of feedback or accumulative scores common to existing systems, trust in a user can be built through their consistent and coherent behaviour on commonfare.net, with actions that align with one's shared values.

From this user feedback, the primary goal of the personal view is to allow individuals to observe their own interactions, and those of others, on commonfare.net. In doing so, related work described in Section 2 supports the use of personal visualisations to motivate further contributions, thereby sustaining the development of commonfare.net. Users can opt to make this contribution visualisation available on their profile, towards establishing trust with other users of the platform. Three potential representations were generated, shown in Figure 6 and compared in a user evaluation described in Section 5. Shared features of each are as follows:

- The date pickers below each visualisation allow a user to view platform activity over time.

- The commonshare value corresponding to the selected date is shown explicitly in the middle of each visualisation.

- Colours used in the visualisations distinguish action types. For example, story-based interactions are dark blue.

While varied in representation, they all allow an interested user to see their activity in different platform areas, and how this contributes to their overall commonshare. The visualisations are interactive, so that users can obtain 'details-on-demand' of specific interactions by hovering over and clicking on different areas of each visualisation.

4.2.1 Use cases. The commonshare representation aims to answer a number of questions users may have about themselves and others:

- Can I trust User X? The commonshare metric is resistant to collusive behaviour. Thus, User Y can view the historic activity of User $\mathrm{X}$ to determine whether User $\mathrm{X}$ is a genuine contributor worth engaging with.

- Is User X like me? To determine whether to make contact with another user, a sense of shared values was seen as important. User Y can interact with the visualisation to see what User X comments on or writes about, and whom User $\mathrm{X}$ is also in contact with

- How much am I contributing? Reputation systems in online platforms are important for motivating fair platform use and cooperation. Users can interact with their personal visualisation to see what they can do to obtain greater value for the platform and themselves.

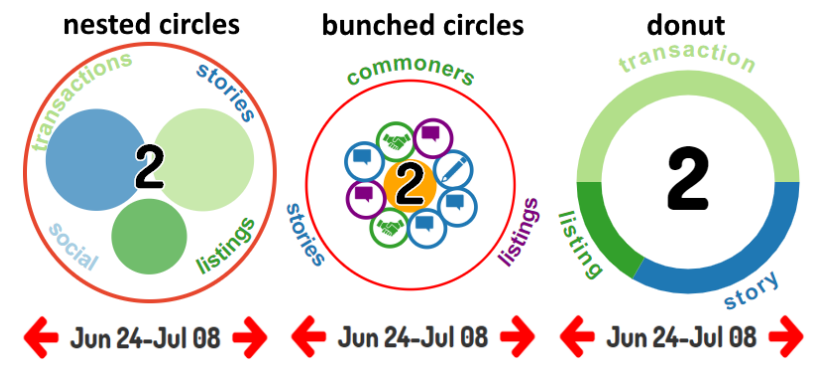

Figure 6: Proposed personal visualisations

\subsection{Public view}

Rather than a tool for analysis, the public view in its current form acts as a dashboard for glanceable monitoring of platform metrics [13]. A key decision was to avoid presenting too many detailed facts and figures without necessary contextual knowledge. Instead, a limited set of simple metrics are provided, to be developed as platform activity increases.

A first version of the dashboard was made available at the end of June 2018, a screenshot of which can be seen in Figure 7. At present, this displays numeric metrics and an interaction graph of the previous two weeks' activity. For the next version, additional simple metrics will be visualised, and more complex indicators crossing two or more metrics - will be defined and implemented.

4.3.1 Use cases. Public engagement in the project is a crucial component for the sustainability and growth of the platform, with use cases of the public dashboard pertaining to two types of organisation: social institutions and research centers. Through contact with institutions - local public authorities in particular - activities on commonfare.net will be promoted in line with their goals regarding welfare policies and self-activation of citizens. Future dashboard developments will enable public authorities to monitor the impact of these activities within the platform. Further, the use of anonymous network analytics data, as provided through an advanced version of the existing dashboard, could be provided in exchange for funds or services offered by research centres. Given the demand for dynamic, labelled network datasets in social network research, the public dashboard will act as an API through which metrics of interest can be accessed by partnering researchers, who in turn can support the sustainability of commonfare.net.

\section{EVALUATION}

As recently conceived components of the platform, the three views are still in iterative development from stakeholder feedback, with evaluation of the network analysis view and the personal view discussed in this section.

\subsection{Network analysis view evaluation}

Following increased platform activity in recent months, it has been possible to view clusters of users and stories that are naturally forming, and determine the perceived accuracy of the commonshare calculation. As partners in the commonfare.net project, our measure of ground truth is our in-depth knowledge of the platform and the members who have been influential in its evolution. By 


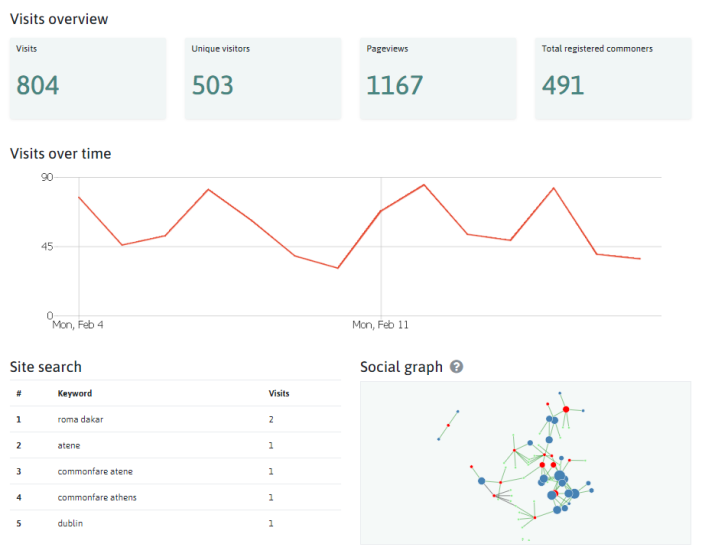

Figure 7: The current "dashboard" public view, with numeric metrics, charts of visits and social activity

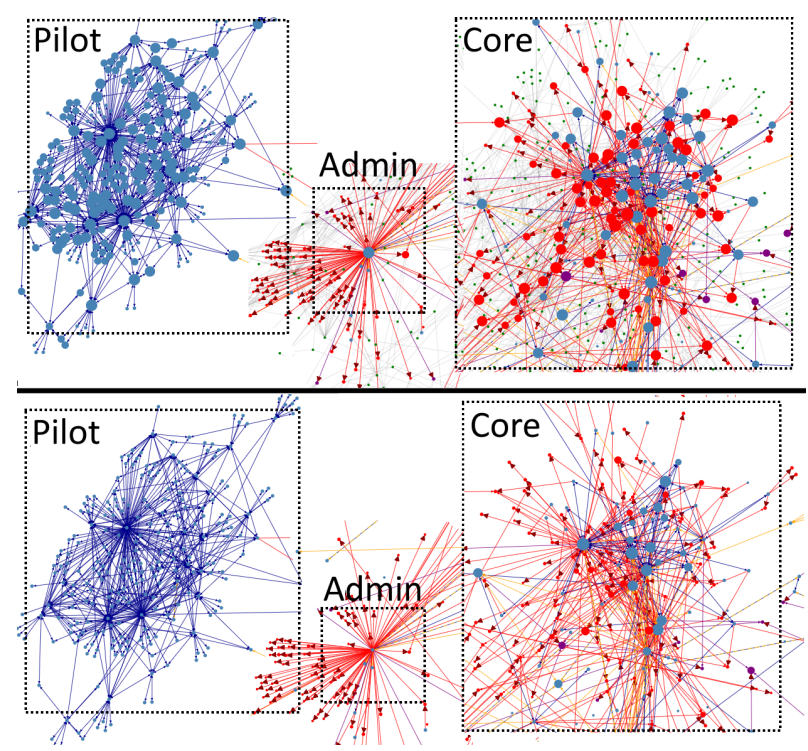

Figure 8: Previous (top) and new interaction graph resulting from recalculation of commonshare

comparing algorithmic output to the 'true' influence of known platform members, we have adjusted parameters to obtain output that accurately reflects members' contributions. Figure 8 illustrates the effects of our adjustment of the weighting algorithm.

Each of the graphs shows the 'overall' commonshare, indicating each node's average contribution over the entire platform history. The Core section highlights the network core, central to most activity on commonfare.net. The Admin section highlights a node with a high degree due to interacting with many otherwise disconnected nodes (in this case, an administrator user who seeded the platform with information posts on public benefits). Finally, the section labelled Pilot represents a week-long pilot conducted in June 2018, where the commonfare.net digital currency was tested at a festival during which purchases could be made from vendors

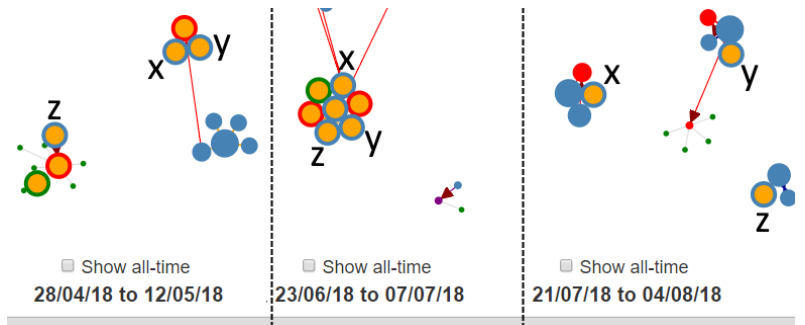

Figure 9: Dynamic community visualisation showing a lack of strong interaction between members

through transactions. The upper graph shows the initial commonshare distribution. It was noted that users who had engaged in the week-long pilot received a disproportionately high commonshare, despite contributing no further platform activity. Also, while the $k$-core algorithm is intended to favour densely connected content, it was noted that users who created content that was not interacted with by other platform members (e.g., the highlighted administrator user) received an inflated commonshare as a result of having a high initial degree. Thus, the network analysis view enabled identification of necessary algorithm adjustments for a fairer allocation of commonshare, as shown in the lower graph.

Insight into an existing platform issue has also been obtained through the dynamic community visualisation, where it has shown that ties on commonfare.net are sporadic and short-lived, indicative of barriers to communication. Figure 9 shows three non-consecutive graph instances in chronological order, grouped by clusters. The leftmost instance shows two users $\mathbf{x}$ and $\mathbf{y}$ who have interacted, separate from a third user $\mathbf{z}$ who exists in a different cluster. Then, two months later (middle instance) the three nodes eventually gather in the same cluster. Following this single instance of community behaviour, no further interaction has occurred between the three, who now exist in separate clusters. As there are no 'friendship' or 'following' connections on commonfare.net, it appears that users become unaware of each other's new content. This insight has motivated the addition of a recommender system to the platform, described in Section 6.2.

\subsection{Personal view evaluation}

Evaluation of the three prototype personal visualisations shown in Figure 6 was undertaken in December 2018. An online survey was implemented using the LimeSurvey platform (www.limesurvey.org), which allowed the visualisations to be integrated into questions.

5.2.1 Survey structure. First, the concept of commonshare was described to users. Each of the three primary types of interactions (conversations, story writing/commenting, transactions) were also described, with emphasis on their significance. Each visualisation was then presented and described in turn, with a multiple-choice question on its content to assess basic understanding. For the main section of the survey, simulated interaction histories for three users were generated as representative examples that the visualisations should be able to distinguish: 
- an inactive user who made one or two interactions in every two-week period, with no preference for types of interactions nor whom they interacted with

- a very active user who consistently had multiple interactions in every two-week period. This user had a clear preference for conversations and transactions with many other users

- a very active user who consistently had multiple interactions in every two-week period, but only with the same two other users (i.e., potentially collusive behaviour)

The three visualisations were presented again in a random order, with each of the three simulated users further randomly paired with a visualisation, in order to minimise potential presentation bias. For each visualisation, participants were asked the following:

- What does this user like to do on commonfare.net? (multiple choice)

- Who does this user interact with? (multiple choice)

- How active is this user on commonfare.net? (multiple choice)

- Based on this commonshare representation, would you have any reason not to trust this user to engage in interactions with them? (yes/no/not sure)

- Please explain your reason for this choice (open-ended)

In total, the survey took approximately 20 minutes to complete. Participants were recruited by publishing a survey link on commonfare.net, requesting feedback from members on improving the platform. No financial incentive was given to complete the survey. In total, 48 responses were received.

5.2.2 Results. Somewhat conflicting results emerged from the survey. While participants unanimously preferred simplicity in representation, the presented information was insufficient to determine whether the simulated user was worthy of trust. Thus, feedback indicated that more detail on interactions would be required to gauge trustworthiness, e.g.: "I usually base my judgement on whether to trust or not someone by relying on more qualitative/descriptive type of feedback"; and "Not enough detail on the content of their interactions to decide their trustworthiness". Participants were able to determine how active a user had been, and the types of interactions they had engaged in, but felt this information to be too simplistic.

To determine what participants would find useful for determining trustworthiness, they were asked to rate eight types of information with 'Yes', 'No' or 'Unsure' as to whether this information would be important. Results are shown in Figure 10, illustrating that, in general, more information of all types is desirable. Participants strongly felt that instances of a user helping another user, and positive/negative feedback from other users, were the most important types of information. In contrast, 'requests for help' were viewed as less indicative of trustworthiness, along with neutral actions such as comments and social interactions.

\subsection{Summary}

Star-based ratings and cumulative feedback scores are popular on e-commerce websites due to their simplicity - allowing them to be quickly read and understood. However, these simplistic metrics only convey a user's reliability to fulfil a transaction or provide a correct answer. In contrast, our results show that a glanceable display of trust is impossible in the context of commonfare.net, whose potential users would instead require rich, in-depth information on other users' history prior to making a decision about them. Although our designs aimed to paint a more detailed picture of a user's interactions, the meaning behind these interactions remains unclear, and thus insufficient to form a reasoned opinion of the user. Schofellen et al. ask "How to visualise the dynamic backstories of an issue in a readable way, in order to engage people in a participatory process around this issue?" [34]. While initial engagement with the personal view is highly likely (it is placed centrally in a user's profile page) its necessarily compact representation conflicts with the need to show these rich "backstories" of interactions.

From the feedback given, participants appreciated a simple, uncluttered visualisation that could be used as a navigation tool for reaching a given interaction, on which further information could then be obtained. This was reflected in one participant's suggestion: "starting representation of donut, but with less clicks: you can use a simple mouseover to enter in the different areas. Or, a click could show you the little circles (shown as in the bunched circles) inside the donut". This strategy of balancing transparency and readability relates to the "staged interaction" suggestion of Schofellen et al., whereby initial readability is prioritised and transparency is introduced gradually, which will guide our subsequent iteration [34].

The majority of participants expressed a preference for the 'donut' visualisation, citing its simplicity and intuitiveness as their reason for this preference, e.g.: "Cleaner and clearer - less busy and therefore less distraction and ambiguity". Conversely, the 'nested circles' and 'bunched circles' were seen as cluttered and complex, despite conveying the same information with similar interactive features. The donut representation is now integrated into user profiles on commonfare.net, with ongoing design work to determine an effective means of providing greater detail on interactions.

\section{DISCUSSION AND ONGOING WORK}

Regarding our research questions outlined in the introduction, our application of social network analysis to the commonfare.net platform data has enabled us to determine a variety of metrics that support our understanding of the community. Foremost, the "commonshare" of platform users serves as a metric of reputation that highlights strong contributors, allowing administrators to understand sources of platform growth through this metric. Towards sustainable community development, administrators could potentially offer these active contributors administrative roles themselves, thereby building a self-sustaining platform. In relation to this, we are also able to detect situations where this metric can be abused through collusion, allowing administrators to take action against behaviour that could damage the community. Finally, our application of dynamic community detection illustrates how relationships may form and dissolve over time on commonfare.net, thereby raising awareness of potential disconnectedness.

As previously discussed, these metrics must be effectively communicated to be of practical value to stakeholders of commonfare.net, motivating our second research question. Addressing this, our community graph-based representation has yielded actionable insights into users' behaviour, particularly regarding the separation of pilot users and low adherence of dynamic communities. These observations have thus motivated investigation into new ways of 


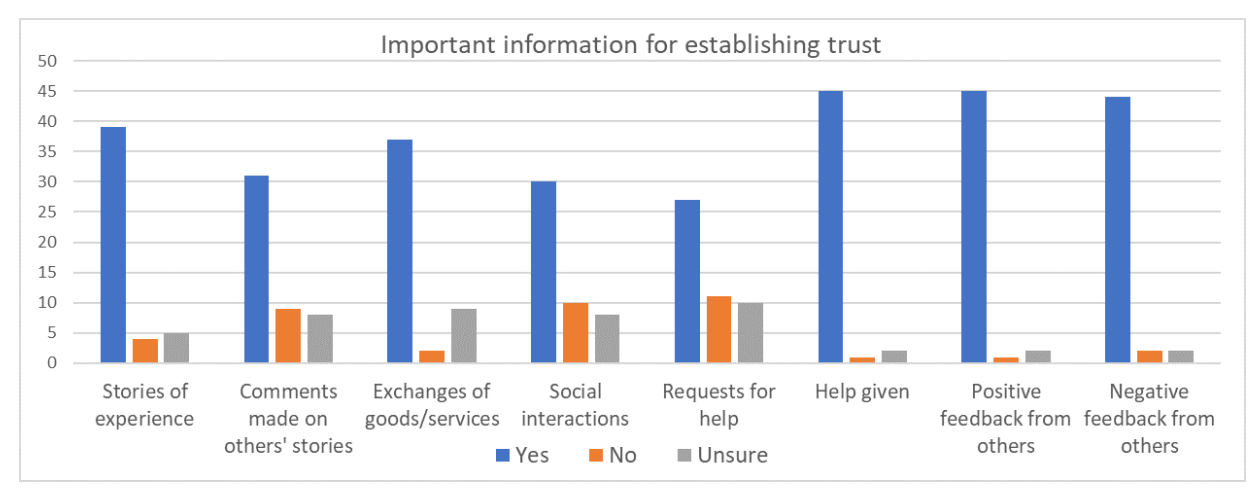

Figure 10: Participants' views on different types of information for establishing trust

encouraging continued use (such as the recommender system described further in this section). Moreover, survey participants in the previous section addressed this question from the perspective of a potential commonfare.net user. From their feedback, it emerged that effective communication of a user's trustworthiness cannot be fulfilled 'at a glance', and instead relies on the ability to find different types of content that the user has created, from which an informed opinion can be made. As such, an effective visualisation must act as a form of menu from which content can be filtered and subsequently accessed.

At the time of writing, a longitudinal evaluation of the efficacy of these visualisations in their context of use is in preparation. Positive metrics such as increased network density or member count could indicate that the inclusion of the views is beneficial, but we also consider more rigorous ways to evaluate their impact.

\subsection{Public view improvements}

Since the beginning of 2019 , increased platform activity by the $400+$ registered users on commonfare.net has initiated plans for publicising this active participation. Discovering the metrics of greatest public interest and how best to communicate these is ongoing work. Participatory design, which has contributed to the evolution of commonfare.net thus far, will now focus on developing the public dashboard, eliciting the views of public institutions and research centres. This will result in an improved dashboard for the final platform release, scheduled for later this year.

\subsection{Recommender system}

Towards encouraging more connections between members, a recommender system has been incorporated into commonfare.net. By providing a given user with recommendations for stories or other users of potential interest, based on previous interactions, this increases the likelihood of their further engagement with the platform.

Our implementation is based on a Personalised PageRank (PPR) algorithm, first described by Haveliwala [21] as an extension of Google's PageRank algorithm [32]. This network-based approach to recommendation mitigates the data sparsity problem experienced in traditional collaborative filtering methods, where there may be insufficient information to determine users' similarity in terms of content they access. Key points of our strategy are as follows:
(1) We use a personalisation vector that assigns an even 'jump' probability to all surrounding nodes of the user and all surrounding nodes of the story they are viewing.

(2) We weight each edge of the entire graph based on its 'age' (i.e., the time of the last interaction) such that newer edges are more likely to be traversed.

(3) In addition to the recommended stories returned from the PPR calculation, we consider 'neglected stories' as those written in the past month that have had little to no interaction.

(4) Users of higher influence in the network (determined by their commonshare) are more likely to be recommended neglected stories, increasing coverage of all stories on the platform, and mitigating potential favouritism bias.

Following the release of the recommender system onto commonfare.net, an empirical evaluation of its effectiveness in sustaining platform engagement will be conducted. Its effect can be determined quantitatively, through observation of activity on the network analysis view and from other engagement metrics obtained through platform analytics. We will use A/B testing, a form of online controlled experiment, in which platform users are randomly split to see no recommendation of further platform content to read (the 'control' condition) or to see these recommendations presented on stories and user profiles (the 'treatment' condition) [12].

\section{CONCLUSION}

The motivation behind our work is that digital communities can benefit from carefully designed visualisations and data analyses, which provide them with knowledge that supports their goals. We designed a set of relevant visualisations based on social network analysis, which are intended to support the specific social innovation goals through a commonfare approach. Fostering commonfare requires users to engage with each other as well as the commonfare.net platform's digital artifacts. To this end, social network analysis allows the identification of emerging patterns of relations, which can be offered to platform members, administrators, and indeed any entity interested in the objectives of the commonfare.net platform. Our work brought us to reflect on the appropriateness of certain visualisations for end-users of a community that may lack technical experience. We also offered community managers tools for investigating hidden patterns of relations, which are not apparent through standard analytics tools. This includes positive patterns 
around the creation of information sub-communities, which could be further supported in achieving their objectives, but also includes negative behaviours such as collusion.

In summary, building and sustaining an online community is a difficult task requiring contributions from all stakeholders. This case study of community visualisations has demonstrated clear benefits in a greater understanding of community behaviour, but also design challenges that remain to be overcome in representing an appropriate level of information to the communities themselves.

\section{ACKNOWLEDGMENTS}

This paper received funding from the European Union's Horizon 2020 research and innovation programme under grant agreement number 687922 . The paper reflects only the authors' views and the Research Executive Agency or European Commission is not responsible for any use that may be made of the information it contains.

\section{REFERENCES}

[1] Mohammad Allahbakhsh, Aleksandar Ignjatovic, Boualem Benatallah, SeyedMehdi-Reza Beheshti, Elisa Bertino, and Norman Foo. 2013. Collusion Detection in Online Rating Systems. In Web Technologies and Applications. Springer Berlin Heidelberg, Berlin, Heidelberg, 196-207.

[2] Bahman Bahmani, Abdur Chowdhury, and Ashish Goel. 2010. Fast Incremental and Personalized PageRank. Proceedings of the VLDB Endowment 4, 3 (Dec 2010), 173-184. https://doi.org/10.14778/1929861.1929864

[3] Vladimir Batagelj and Matjaž Zaveršnik. 2011. Fast algorithms for determining (generalized) core groups in social networks. Advances in Data Analysis and Classification 5, 2 (2011), 129-145.

[4] Francesco Bellini, Antonella Passani, Marina Klitsi, and Wim Vanobberger. 2016 Exploring impacts of collective awareness platforms for sustainability and social innovation. Eurokleis Press, Rome, Italy.

[5] Vincent D Blondel, Jean Loup Guillaume, Renaud Lambiotte, and Etienne Lefebvre 2008. Fast unfolding of communities in large networks. Fournal of Statistical Mechanics: Theory and Experiment 2008, 10 (Oct 2008), P10008. https://doi.org/ $10.1088 / 1742-5468 / 2008 / 10 / \mathrm{P} 10008$

[6] Francesco Botto and Maurizio Teli. 2017. PIE News. A public design project toward commonfare. The fournal of Community Informatics 13, 2 (Aug. 2017), 87-105. http://www.ci-journal.net/index.php/ciej/article/view/1383

[7] Phil E Brown and Junlan Feng. 2011. Measuring user influence on twitter using modified k-shell decomposition. In The Social Mobile Web, Papers from the 2011 ICWSM Workshop. AAAI, Barcelona, Catalonia, Spain, 18-23.

[8] Cen Cao, Qingjian Ni, and Yuqing Zhai. 2015. An improved collaborative filtering recommendation algorithm based on community detection in social networks. In Proceedings of the 2015 Annual Conference on Genetic and Evolutionary Computation. ACM Press, New York, USA, 1-8. https://doi.org/10.1145/2739480.2754670

[9] European Commission. 2010. Empowering People, Driving Change - Social Innovation in the European Union. (2010). Retrieved January 25th, 2019 from http://ec.europa.eu/DocsRoom/documents/13402/attachments/1/translations

[10] European Commission DG CONNECT. 2018. Collective Awareness Platforms for Sustainability and Social Innovation. (2018). Retrieved January 25th, 2019 from https://ec.europa.eu/digital-single-market/en/collective-awareness

[11] Shane Dawson. 2010. 'Seeing' the learning community: An exploration of the development of a resource for monitoring online student networking. British Journal of Educational Technology 41, 5 (Sep 2010), 736-752. https://doi.org/10. $1111 / \mathrm{j} .1467-8535.2009 .00970 . \mathrm{x}$

[12] Eleri Dixon, Emily Enos, and Scott Brodmerkle. 2011. A/B testing of a webpage. (Jul 2011). US Patent 7,975,000.

[13] Stephen Few. 2013. Information dashboard design (2nd ed.). Analytics Press, Burlingame, CA, USA.

[14] Santo Fortunato. 2010. Community detection in graphs. Physics Reports 486, 3-5 (Feb 2010), 75-174. https://doi.org/10.1016/J.PHYSREP.2009.11.002

[15] Andrea Fumagalli and Stefano Lucarelli. 2015. Finance, austerity and commonfare. Theory, Culture \& Society 32, 7-8 (2015), 51-65.

[16] Antonios Garas, Frank Schweitzer, and Shlomo Havlin. 2012. A k-shell decomposition method for weighted networks. New fournal of Physics 14, 8 (Aug 2012), 083030. https://doi.org/10.1088/1367-2630/14/8/083030

[17] Gian-Luca Gasparini, Aurora Prospero, Aikaterini Katmada, Anna Satsiou, Miroslav Konecny, and Peter Hanečák. 2018. Technology Adoption and Social Innovation: Assessing an Online Financial Awareness Platform. In Internet
Science. Springer International Publishing, Cham, 21-30.

[18] Sohaib Ghani, Bum Chul Kwon, Seungyoon Lee, Ji Soo Yi, and Niklas Elmqvist. 2013. Visual analytics for multimodal social network analysis: a design study with social scientists. IEEE Transactions on Visualization and Computer Graphics 19, 12 (Dec 2013), 2032-41.

[19] Eric Gilbert and Karrie Karahalios. 2009. Using social visualization to motivate social production. IEEE Transactions on Multimedia 11, 3 (2009), 413-421.

[20] Derek Greene, Donal Doyle, and Padraig Cunningham. 2010. Tracking the Evolution of Communities in Dynamic Social Networks. In Proceedings of the 2010 International Conference on Advances in Social Networks Analysis and Mining. IEEE, Washington, DC, USA, 176-183. https://doi.org/10.1109/ASONAM.2010.17

[21] Taher H. Haveliwala. 2002. Topic-sensitive PageRank. In Proceedings of the 11th International Conference on World Wide Web (WWW'02). ACM, New York, NY, USA, 517-526. https://doi.org/10.1145/511446.511513

[22] Jeffrey Heer and Danah Boyd. 2005. Vizster: Visualizing online social networks. In Proceedings - IEEE Symposium on Information Visualization, INFO VIS. IEEE, Minneapolis, MN, USA, 33-40.

[23] Paul Jaccard. 1912. The Distribution of the Flora in the Alpine Zone. New Phytologist 11, 2 (1912), 37-50. https://doi.org/10.1111/j.1469-8137.1912.tb05611.x

[24] Thomas Kelder, Martijn P. Van Iersel, Kristina Hanspers, Martina Kutmon, Bruce R Conklin, Chris T Evelo, and Alexander R Pico. 2012. WikiPathways: Building research communities on biological pathways. Nucleic Acids Research 40, D1 (Jan 2012), D1301-7. https://doi.org/10.1093/nar/gkr1074

[25] David Kempe, Jon Kleinberg, and Éva Tardos. 2005. Influential Nodes in a Diffusion Model for Social Networks. In Proceedings of the 32nd International Conference on Automata, Languages and Programming (ICALP'05). Springer-Verlag, Berlin, Heidelberg, 1127-1138.

[26] Maksim Kitsak, Lazaros K. Gallos, Shlomo Havlin, Fredrik Liljeros, Lev Muchnik, H. Eugene Stanley, and Hernán A. Makse. 2010. Identification of influential spreaders in complex networks. Nature Physics 6, 11 (Nov 2010), 888-893. https: //doi.org/10.1038/nphys1746

[27] S. Mckenna, D. Staheli, and M. Meyer. 2015. Unlocking user-centered design methods for building cyber security visualizations. In 2015 IEEE Symposium on Visualization for Cyber Security (VizSec). IEEE, Chicago, IL, USA, 1-8. https: //doi.org/10.1109/VIZSEC.2015.7312771

[28] Nicola Morelli, Ingrid Mulder, Grazia Concilio, Janice S. Pedersen, Tomasz Jaskiewicz, Amalia de Götzen, and Marc Arguillar. 2017. Open Data as a New Commons. Empowering Citizens to Make Meaningful Use of a New Resource. In Internet Science. Springer International Publishing, Cham, 212-221.

[29] J. L.. Moreno. 1934. Who shall survive?: A new approach to the problem of human interrelations. Nervous and Mental Disease Publishing Co, Washington DC, USA, xvi, 441. https://doi.org/10.1037/10648-000

[30] M. E. J. Newman. 2003. The Structure and Function of Complex Networks. SIAM Review 45, 2 (Jan 2003), 167-256.

[31] Chris North. 2006. Toward measuring visualization insight. IEEE Computer Graphics and Applications 26, 3 (May 2006), 6-9.

[32] Lawrence Page, Sergey Brin, Rajeev Motwani, and Terry Winograd. 1999. The PageRank Citation Ranking: Bringing Order to the Web. Technical Report 1999-66. Stanford InfoLab. http://ilpubs.stanford.edu:8090/422/

[33] Symeon Papadopoulos, Yiannis Kompatsiaris, Athena Vakali, and Ploutarchos Spyridonos. 2012. Community detection in Social Media. Data Mining and Knowledge Discovery 24, 3 (May 2012), 515-554. https://doi.org/10.1007/ s10618-011-0224-z

[34] Jessica Schoffelen, Sandy Claes, Liesbeth Huybrechts, Sarah Martens, Alvin Chua, and Andrew Vande Moere. 2015. Visualising things. Perspectives on how to make things public through visualisation. CoDesign 11, 3-4 (2015), 179-192. https://doi.org/10.1080/15710882.2015.1081240

[35] Haiying Shen, Yuhua Lin, Karan Sapra, and Ze Li. 2016. Enhancing Collusion Resilience in Reputation Systems. IEEE Transactions on Parallel and Distributed Systems 27, 8 (Aug 2016), 2274-2287.

[36] Lingling Sun and Julita Vassileva. 2006. Social Visualization Encouraging Participation in Online Communities. In Groupware: Design, Implementation, and Use. Springer Berlin Heidelberg, Berlin, Heidelberg, 349-363.

[37] Nina Valkanova, Sergi Jorda, Martin Tomitsch, and Andrew Vande Moere. 2013. Reveal-it!: The Impact of a Social Visualization Projection on Public Awareness and Discourse. In Proceedings of the SIGCHI Conference on Human Factors in Computing Systems (CHI '13). ACM, New York, NY, USA, 3461-3470.

[38] Fernanda B. Viégas, Scott Golder, and Judith Donath. 2006. Visualizing Email Content: Portraying Relationships from Conversational Histories. In Proceedings of the SIGCHI Conference on Human Factors in Computing Systems (CHI '06). ACM, New York, NY, USA, 979-988. https://doi.org/10.1145/1124772.1124919

[39] Stanley Wasserman and K L M Faust. 1994. Social Network Analysis: Methods and Applications. Cambridge University Press, Cambridge, UK. 825 pages.

[40] Yingcai Wu, Nan Cao, David Gotz, Yap Peng Tan, and Daniel A. Keim. 2016. A Survey on Visual Analytics of Social Media Data. IEEE Transactions on Multimedia 18, 11 (Nov 2016), 2135-2148. https://doi.org/10.1109/TMM.2016.2614220 